\title{
A WAVELET BASED MAMMOGRAPHIC SYSTEM
}

\author{
Andrew Laine 1 \\ Michael Lewis ${ }^{2}$ \\ Fred Taylor $^{1}$
}

(1) The University of Florida, Gainesville, FL, USA

(2) The Athena Group Inc., Gainesville, FL, USA.

\begin{abstract}
Mammography's role in the ABSTRACT: detection of breast cancer at early stages is well known. Although more accurate than other existing techniques, mammography still only finds 80 to 90 percent of breast cancers. It has been suggested that mammograms, as normally viewed, display only about $3 \%$ of the total information detected. The general inability to detect small tumors and other salient features within mammograms motivates our investigation of a system we call the Mammogram Display System [MDS]. The core technology used for MDS image enhancement is the wavelet transform.
\end{abstract}

Introduction: The MDS is being developed for the purpose of enhancing a radiologist's perception of mammographic features (including lesions, masses, and calcifications) and contribute towards a more accurate diagnosis. The MDS will increase the chances of early detection of breast cancers (improved quality) and, for most patients, will shorten the mammogram evaluation time (lowered costs). The analysis and display mammogram features is accomplished through the use of wavelet ${ }^{1}$ transforms (multi-resolution) and a synergistic implementation technology. Preliminary studies have shown that the MDS can make unseen or barely seen features of a

1 Wavelet transforms are a relatively new innovation that permit both global and local signal and image representations in the time and frequency domains. Wavelets are used to decompose an image into a set of frequency channels having a constant bandwidth on a logarithmic scale. mammogram more obvious without additional radiation. This, in a sense, could have been predicted a priori since multiresolution image processing is a known biological mechanism in primate vision. The multiresolution decomposition of wavelet transforms provides a natural hierarchy in which to embed an interactive paradigm for mammogram analysis. With the MDS the radiologist may first choose to look for coarse features (e.g., dominant mass) and later examine finer features (e.g., microcalcifications). The MDS can produce significantly enhanced mammographic images which complement existing modalities, allow a radiologist to interactively examine features within a selected scale space, and guide the radiologist to suspicious locations. The image processing advantages have a price; that price is a high-computation burden. The technical narrative will also show how this problem can be mitigated with our proposed advanced implementation strategy. The hardware embodiment of the MDS is based on a new class of high-speed arithmetic processor.

SIGNIFICANCE Many cancers escape density of surrounding

breast tissue. Since differences in attenuation of the various soft tissue structures in the female breast are small, it is necessary to use low levels of $X$-ray energy to obtain high contrast in mammographic film. Furthermore, the contrast between the layers of soft tissues of the breast is inherently low, and relatively minor changes in mammary structure can signify the presence of a malignant breast tumor. The detection is more exasperating in mammography than in most other forms of radiography. The radiologist must search for malignancies such as microcalcifications, dominant and stellate masses, as well as textures of fibrous tissues. 
The diagnosis of breast cancer at an early stage is critical to patient survival. The cure rate for breast lesions of $0.5 \mathrm{~cm}$ or less in diameter can approach $100 \%$. Despite advances and improvements in mammography and mammogram screening, the detection of early breast cancer (those cancers $1.0 \mathrm{~cm}$ or less in diameter) remains difficult. Unfortunately, a primary breast carcinoma can metastasize when it consists of a relatively small number of cells, far below our present threshold of detection. The current limitation of mammography to detect all breast cancers motivates our investigation of wavelet methods of analysis for digital mammography.

The literature is replete with image processing techniques for the enhancement of mammograms. Algorithms based on adaptive neighborhood image processing of selected features have been investigated. Other methods introduced noise and/or digitization effects at the point that the desired features were lost. Included in this mix are adaptive neighborhood techniques that utilized lowlevel image analysis and knowledge about a desired feature to identify an enhancement function which improves the contrast of specific features. The later was developed as a method which defined a global model to compute the best contrast enhancement function based on a priori knowledge of specific types of mammographic features. Researchers have found, however, that a suitable contrast function, while important to enhancement process, is difficult to select.

Method have been developed for the enhancement of chest and breast radiographs by using spatial filtering with the linear combination of an original image and two smoothed images obtained from the original image by applying different spatial masks. The process was completed by nonlinear contrast stretching. This method enhanced edges while minimally amplifying noise. Other recent approaches to automate the detection of tumors in mammograms have relied heavily on some method of enhancing features. In the development of a "smart" workstation for mammography, using linear and nonlinear filters in a difference-image approach in order to increase the signal-to-noise ratio of micro calcifications. Another development is an automated system for the detection and classification of particular types of tumors in digitized mammograms. A threshold parameter and "fuzzy" pyramid links identified homogeneous regions while a second threshold parameter was applied to separate selected regions (tumors) from the background. Others compared several image enhancement methods for detecting circumscribed masses in mammograms. The method compared an edge-preserving smoothing function, a halfneighborhood, nearest neighborhood, directional smoothing, and median filtering, and then proposed a method for selective median filtering.

Despite these accomplishments, enhancement of mammographic features remains a troublesome task for traditional digital image processing techniques. We describe what we believe to be an enhanced digital mammogram display paradigm which has the ability to provide radiologists with the technology to make early detection of breast cancers reliably. However, this by itself would not necessarily improve the practice of mammography unless it could be implemented in high-resolution, low-cost, real-time (or near real-time) hardware. The implementation issues are equally challenging. The paper narrative will provide technical insights into the technology used to design an MDS prototype which currently undergoing testing.

\section{DESIGN AND METHODS:}

Preliminary studies have shown that our method can make unseen or barely seen features of a mammogram more obvious without requiring additional radiation. To date, our study has examined the reconstruction of mammograms from several analyzing (wavelet) functions. In our preliminary study, a cubic spline analyzing wavelet was used to accomplish each reconstruction. The original (dense) mammogram and an enhancement are shown in Figure 1. In Figure 2(a) we show the magnitude of the waveiet coefficients in the horizontal (left) and vertical (right) directions over the first three levels of the transform. Figure 2(b) displays the combined horizontal and vertical coefficients (left), direction (gradient) of the coefficients (center), and the wavelet maxima (right). Note how the geometric shape of the calcifications can be readily seen in the left and center columns for the finer scale sizes. The wavelet maxima [right] can be used to isolate features within a given level of the transform. 

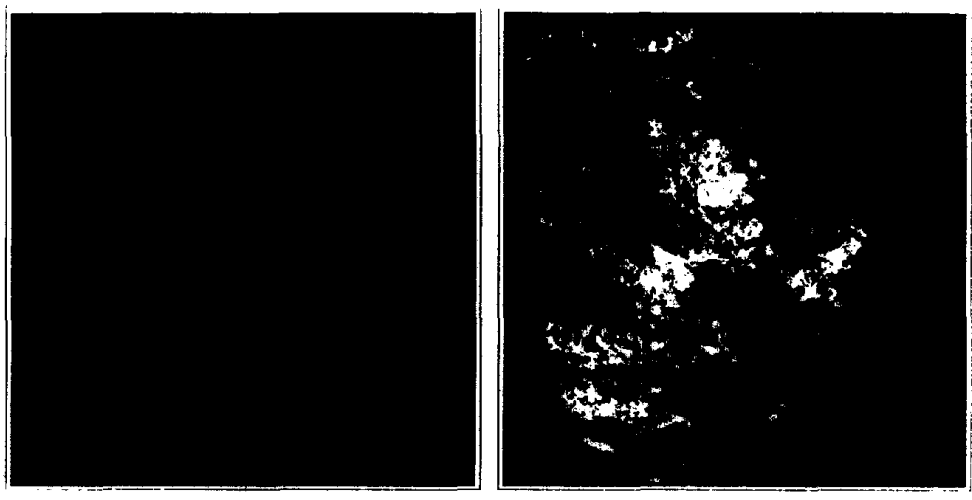

Figure 1: Original dense mammogram (left) and enhanced mammogram (left)

To test whether the proposed method can enhance pathologically meaningful shapes from a mammogram, a mathematically defined pattern consisting of regular and irregular shapes (simulating microcalcifications) were superimposed on a mammogram. The processed database produced an enhanced image which was examined. The results, which cannot be done justice with a photocopy, did convince us that the introduced pattern, which could not be easily seen in the superimposed image, was highly recognizable in the enhanced image.

The experimental latency, on a Sun Workstation, was long. Detection of microcalcifications in breast cancer diagnosis suggests the need for large matrix sizes. However, larger matrix sizes require high resolution digitizers, greater computer storage capacities and more powerful computers. The importance of matrix size in wavelet processing was experimentally observed. Digitization "spot sizes" of $35 \mu \mathrm{m}$ (assuming $7 \mathrm{k} \times 6 \mathrm{k}$ matrix size for an 8"x10" film size), $70 \mu \mathrm{m}, 100 \mu \mathrm{m}$, and $200 \mu \mathrm{m}$ are assumed. Thus, a conventional mammogram could range in size from $2.5 \mathrm{MByte}$ up to $84 \mathrm{MByte}$, for 16-bit ( 2 byte) pixels. In the future, as the image becomes larger and of higher resolution (tending to $2.5 \mathrm{Gbit}$ ), the computational issue will become significantly more important.

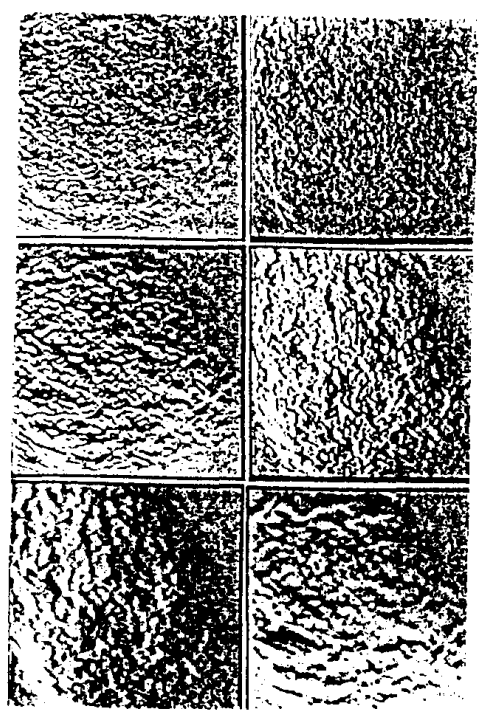

(a) Horizontal and vertical wavelets
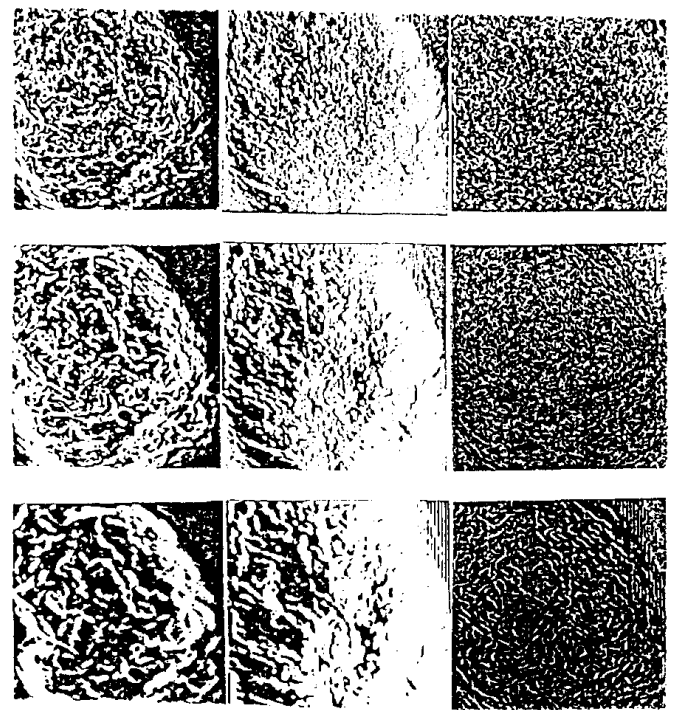

(b) Composite, directional (grad.), and maxima

Figure 2: Wavelet analysis of a mammogram 
Our technology is based on the application of the residue number system [RNS] and in particular the patent pending Logarithmic RNS (LRNS). The A+ technology is unique to Athena and has been developed as a basic FIR device under a DOD SBIR award. This provides a basic building block for the study of wavelet transforms and ANNs. Our study will concentrate on the wavelet processor. There is, at this time, little available in the area of wavelet ASIC processors. For example, Aware's Wavelet Transform Processor (WTP) has been available since 1991. It is compared to the proposed A+ chip in Table 1

\begin{tabular}{|c|c|}
\hline WTP & Athena chip \\
\hline 16-bit inputs and outputs & $\leq 24$-bit inputs and outputs \\
\hline 2,4 , or 6 coefficients & up to 16 coefficients \\
\hline forwardlreverse WT & forwardlreverse WT \\
\hline Pipeline latency $=10 \mathrm{clk}$ & Pipeline latency $=1 \mathrm{clk}$ \\
\hline $30 \mathrm{HMz}$ clock & $50 \mathrm{MHz}$ clock \\
\hline
\end{tabular}

\section{LITERATURE CITED}

Mammographic Image Processing

[Bra91] Brzakvoic, D., X. M. Luo and P. Brzakvoic, "An approach to automated detection of tumors in mammograms", IEEE Trans. Med. Imaging, Vol. MI-9, No. 3, 233-241, 1991

[Dha87] Dhawan, A. P. and Gordon, R., Reply to comments on "Enhancement of mammographic feature by optimal adaptive neighborhood image processing", IEEE Trans. Med. Imaging, MI-6, 82, 1987.

[Dha88] Dhawan, A. P., and Le Royer, E., "Mammographic feature enhancement by computerized image processing", Comput. Methods Programs Biomed., 1988

[Dha86] Dhawan, A. P., Buelloni, G., and Gordon, $R$. "Enhancement of mammographic feature by optimal adaptive neighborhood image processing", IEEE Tran. Med. Imaging, MI-5, 8, 1986. (Errata: MI-5, 1986).

[Gig91] Giger, M.L., et. al., "Development of a "smart" workstation for use in mammography", SPIE Vol. 1445 Image Processing, 1991.

[Goi83] Goin, J. E. and Haberman, J. D., "Automated breast cancer detection by thermography: performance goal and diagnostic feature identification", Pattern Recog., 16, 125, 1983.

[Ros76] Rosenfield, A. and A.C. Kak, Digital Image Processing, NY: Academic Press, 1976

\section{WAVELETS}

[Dau90] Daubechies I., "The wavelet transform, timefrequency localization and signal analysis", IEEE Trans. on Info. Theory, Sept., 1990.

[Lai91] Laine, A., and G.-C. Roman, "A Parallel Algorithm for Incremental Stereo Matching Using Geometric Constraints", to appear in the IEEE Transactions on Robotics and Automation, February, 1991.

[Lem86] Lemarie, P. G. and Y. Meyer, "Ondelettes et bases Hilbertiennes," Revista Mathematica Ibero Americana, vol. 2, 1986.

[Rio91] Rioul, O., "Fast Algorithms for the Continuous Wavelet Transform", IEEE ASSP Conference, Toronto, May 1991.

\section{RESIDUE NUMBER SYSTEM}

[IEE86] Soderstrand, M., Jenkins, W., Jullien, G., Taylor, F., ed., Residue Number System Arithmetic: Modern Applications In Digital Signal Processing, IEEE Press, 1986.

[Zel91] Zelniker, G. and Taylor, F., "A Simplified Finite Field ALU for the Quadratic Residue Number System", IEEE Trans. on Ckts. and Sys., Dec. 1991

This work was supported, in part, under an NIH Phase I SBIR award. NIH 1-R43-CA5-0232-01. 